\title{
Validation of a next-generation sequencing (NGS) panel to improve the diagnosis of X-linked hypophosphataemia (XLH) and other genetic disorders of renal phosphate wasting
}

\author{
Susanne Thiele', Ralf Werner ${ }^{1,2}$, Annika Stubbe ${ }^{3}$, Olaf Hiort ${ }^{1}$ and \\ Wolfgang Hoeppner ${ }^{3,4}$
}

${ }^{1}$ Division of Paediatric Endocrinology and Diabetes, Department of Pediatrics, University of Lübeck, Lübeck, Germany, ${ }^{2}$ Institute for Molecular Medicine, University of Lübeck, Lübeck, Germany, ${ }^{3}$ Labor Dr. Heidrich und Kollegen MVZ GmbH, Hamburg, Germany, and ${ }^{4}$ Bioglobe GmbH, Hamburg, Germany

Correspondence should be addressed to O Hiort

Email

Olaf.Hiort@uksh.de

\section{Abstract}

Background: Hypophosphataemic rickets (HR) comprise a clinically and genetically heterogeneous group of conditions, defined by renal-tubular phosphate wasting and consecutive loss of bone mineralisation. X-linked hypophosphataemia $(\mathrm{XLH})$ is the most common form, caused by inactivating dominant mutations in PHEX, a gene encompassing 22 exons located at Xp22.1. XLH is treatable by anti-Fibroblast Growth Factor 23 antibody, while for other forms of HR such as therapy may not be indicated. Therefore, a genetic differentiation of HR is recommended.

Objective: To develop and validate a next-generation sequencing panel for HR with special focus on PHEX. Design and methods: We designed an AmpliSeq gene panel for the IonTorrent PGM next-generation platform for PHEX and ten other HR-related genes. For validation of PHEX sequencing 50 DNA-samples from XLH-patients, in whom 42 different mutations in PHEX and 1 structural variation have been proven before, were blinded, anonymised and investigated with the NGS panel. In addition, we analyzed one known homozygous DMP1 mutation and two samples of HR-patients, where no pathogenic PHEX mutation had been detected by conventional sequencing.

Results: The panel detected all 42 pathogenic missense/nonsense/splice-site/indel PHEX-mutations and in one the known homozygous DMP1 mutation. In the remaining two patients, we revealed a somatic mosaicism of a PHEX mutation in one; as well as two variations in DMP1 and a very rare compound heterozygous variation in ENPP1 in the second patient.

Conclusions: This developed NGS panel is a reliable tool with high sensitivity and specificity for the diagnosis of XLH and related forms of HR.

\section{Introduction}

X-linked hypophosphataemia (XLH) (OMIM\#307800) is the most common genetic disorder of phosphate homeostasis characterized by renal phosphate wasting and hypophosphataemia. It affects about one in 20000 individuals (1) and follows an X-chromosomal dominant inheritance pattern. Printed in Great Britain
Children affected by XLH present with a broad phenotypic spectrum ranging from isolated hypophosphatemia with few clinical signs up to severe symptoms, such as rickets with extreme lower limb deformities, distinct tooth problems (such as dental abscesses), and a disproportionate short stature 
(reviewed in (2)). In adulthood, further symptoms may occur, such as osteomalacia, arthrosis, pseudo fractures, and diminished final height (3). Further clinical signs are hearing difficulties, enthesopathy, and muscular dysfunction. As the disorder is more under focus during the last few years, more clinical signs have been associated with XLH, such as Arnold-Chiari malformation and other craniofacial abnormalities. Furthermore, bone pain is a very pronounced sign in XLH, leading to an impairment of the quality of life in affected children and adults (reviewed in (2)).

XLH is caused by mutations in the PHEX gene encoding the cleavage enzyme phosphate-regulating neutral endopeptidase (PHEX) located on the $\mathrm{X}$ chromosome. Today, more than 588 mutations have been reported, spread all over the 22 exons of the PHEX gene (http://www. hgmd.cf.ac.uk/). This includes point mutations, deletions, insertions, as well as intronic variations, presumably altering PHEX function. Although the pathophysiology of $\mathrm{XLH}$ is not fully understood, the inactivation of the PHEX protein (expressed predominantly in osteoblasts) leads to an increase of fibroblasts growth factor 23 (FGF23) levels. High FGF23 levels cause urinary phosphate wasting by down-regulating the renal sodium phosphate transporters 2a and 2c ( NaPi2a and $\mathrm{NaPi} 2 \mathrm{c}$, respectively) and reducing transformation of 25-OH-Vitamin $\mathrm{D}_{3}$ to the active vitamin $\mathrm{D}$ form $1,25-\mathrm{OH}_{2}$-Vitamin $\mathrm{D}_{3}(4,5,6)$ leading to abnormal low levels of $1,25-\mathrm{OH}_{2}$-Vitamin $\mathrm{D}_{3}$ despite of hypophosphatemia. Therefore, laboratory hallmarks in XLH are hypophosphataemia, reduced renal-tubular phosphate reabsorption, and inappropriately low to normal 1,25 $(\mathrm{OH})_{2}$-vitamin $\mathrm{D}_{3}$ levels. In addition, elevated alkaline phosphatase is seen as a marker of higher bone turnover related to rickets.

There is a high overlap between XLH and other forms of HR both in clinical as well as in laboratory findings. Some of those components that have been associated with elevated FGF23 expression or decreased degradation of FGF23 include (amongst others) FAM20C (family with sequence similarity 20 member C) (7), furthermore, ENPP1 (ectonucleotide pyrophosphatase/phosphodiesterase) encoded by the ENPP1 gene (8), and DMP1 (dentin matrix acidic phosphoprotein 1) encoded by the DMP1 gene (9, 10). Consequently, inactivating mutations in these genes also lead to an elevation of serum FGF23 levels and to disorders with a similar phenotype to XLH. A further known pathomechanism is caused by increased FGF23 levels due mutations in FGF23 itself, which affect the cleavage site for degradation. This condition follows an autosomal-dominant (AD) inheritance and the phenotype seems to be milder (11). In contrast to XLH, ADHR shows incomplete penetrance, variable age at onset, and vanishing of the phosphate-wasting defect in rare cases $(11,12,13)$. Table 1 summarizes different forms of HR with their biochemical characteristics in comparison to nutritional rickets.

Conventional therapy of HR includes oral phosphate supplementation and, in forms with FGF23-mediated hypophosphatemia, calcitriol; however, this therapy further stimulates FGF23 excretion, enforcing the renal phosphate wasting (6). Recently, a novel therapy with an anti-FGF23 antibody has been approved and current results demonstrate an enormous impact on medical outcome for patients with XLH in children $(14,15,16)$ and adults (17). The novel therapy with Burosumab has only been approved for XLH, and it is currently unknown if patients with other forms of HR with FGF23 elevation might profit or not from this therapy. Some forms of HR, for example, caused by ENPP1 mutation, may even have unfavorable effects from Burosumab such as hypercalcemia and calcification, although this is also currently unknown.

In XLH, early diagnosis followed by an immediate treatment has a strong impact on the patient's longterm outcome (18). However, the diagnosis of this rare condition is often delayed. The first clinical signs are often mild and occur when a toddler starts standing alone and walking, leading to bowing of the lower limbs, which can also be the first sign of rickets due to vitamin D deficiency. Even the biochemical signs are not always straight forward and may altogether not differentiate between XLH and other forms of HR (19). Therefore, the molecular genetic confirmation of the clinically and biochemically based diagnosis of XLH and differentiation from other forms of HR has been recommended by many specialists of this disorder (2). Until today, the gold standard for the search of mutations in the PHEX gene has been Sanger sequencing of all 22 exons, including the exon/intron boundaries for detecting splice site mutations, followed by multiplex ligation-mediated probe amplification analysis (MLPA). By this approach, the diagnosis is relatively expensive and time-consuming and cannot detect other forms of HR. If several patients were to be analyzed in one approach and several genes of one panel were to be examined for some of the patients, we were able to determine a total time and cost-saving from 10 samples onward by NGS analysis.

For these reasons, we developed a next-generation sequencing (NGS) panel comprising not only all exons and the intron boundaries of the PHEX gene but also ten other genes, in which mutations are known to cause renal 
$|\mathrm{e}| \leftarrow \leftarrow \leftarrow \leftarrow \leftarrow$

$\mid \begin{array}{llllll}\mathbf{I} & 1 & 1 & 1 & 1 & \rightarrow\end{array}$

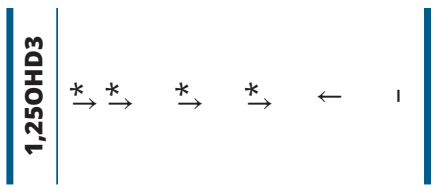

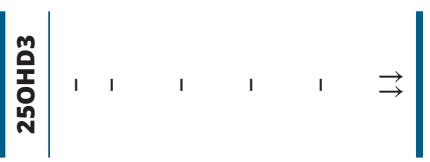

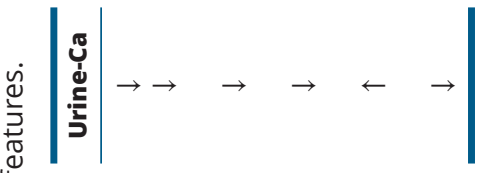

phosphate wasting disorders to ensure the distinct differential diagnosis of XLH.

\section{Methods}

The ethical committee of the University of Lübeck approved this part of the study in January 2004 (04-020) and confirmed the ethical correctness for developing of a NGS panel with the same samples in October 2018 (18-271).

\section{DNA samples with known PHEX-mutations}

For validation of the gene panel, we involved DNA samples from $50 \mathrm{XLH}$ patients with a confirmed clinical, biochemical, and molecular genetic diagnosis representing 42 different PHEX mutations. The mutations cover a broad spectrum of short deletions, missense, nonsense and splice site mutations covering most of the exons of the PHEX gene, as well as one large duplication of exon 12. The samples were anonymized and blinded before inclusion into the panel investigation. DNA had been prepared from EDTA-blood with Qiagen blood kit (Qiagen).

After validation of the gene panel for PHEX, we analyzed three additional samples in which HR had been diagnosed by clinical and biochemical signs in the patients, but without a detectable mutation in PHEX analyzed by Sanger sequencing. One sample has a known homozygous variant in the DMP-1 gene. All 53 samples were anonymized with continuous numbers, only harboring the information of the sex chromosomes differentiation between male and female samples.

\section{Establishing the IonTorrent AmpliSeq gene panel}

We utilized the IonTorrent PGM next-generation platform (Thermo Fisher Scientific) in our setting for molecular diagnosis of XLH. The NGS was performed according to the standard protocol recommended by the system supplier.

The gene panel was designed using the AmpliSeq Designer online tool from Thermo Fisher Scientific (https://www.ampliseq.com/). Technical characteristics of the gene panel are shown in Table 2 and Supplementary Table 1 (see section on supplementary materials given at the end of this article). Patient DNA was amplified by multiplex PCR. We included 11 genes in our gene panel of HR-related disorders - PHEX, FGF23, DMP1, ENPP1, 
Table 2 Technical characteristics of the next-generation sequencing (NGS) panel for X-linked hypophosphataemia.

\begin{tabular}{l}
\hline \\
\hline Genes \\
PHEX \\
CLCN5 \\
DMP1 \\
ENPP1 \\
FAM20C \\
FGF23 \\
FGFR1 \\
KL \\
SLC34A1 \\
SLC34A3 \\
SLC9A3R1 \\
NGS Panel details \\
Number of genes \\
Panel size \\
Primer Pools \\
Total number of exons \\
Total number of amplicons \\
Amplicon lengths \\
Coverage
\end{tabular}

\begin{tabular}{l}
\hline Ref Seq \\
\hline NM_000444.5 \\
NM_000084.,4 \\
NM_004407.3 \\
NM_006208.2 \\
NM_020223.3 \\
NM_020638.2 \\
NM_023110.2 \\
NM_004795.3 \\
NM_003052.4 \\
NM_080877.2 \\
NM_004252.4
\end{tabular}

\begin{tabular}{c}
\hline Number of exons \\
\hline 22 \\
11 \\
5 \\
25 \\
10 \\
3 \\
17 \\
5 \\
12 \\
12 \\
6
\end{tabular}

Number of homopolymers

Characteristics

\begin{tabular}{c}
11 \\
$45.98 \mathrm{~kb}$ \\
2 \\
128 \\
245 \\
$125-275 \mathrm{bp}$ \\
$99.88 \%$ \\
\hline
\end{tabular}

SLC34A3, CLCN5, SLC34A1, SLC9A3R1, FAM2OC, FGFR1 and $K L-$ which are involved in phosphate metabolism or are known to cause different types of HR. Sequence analysis was carried out with the software module SeqNext (SeqPilot ${ }^{\mathrm{TM}}$, JSI, Ettenheim, Germany). Small gaps in the designed panel, mainly due to large homopolymer stretches, were complemented by Sanger sequencing (Table 2 and Supplementary Tables 2, 3).

\section{Multiplex ligation-mediated probe amplification (MLPA)}

Large deletions or duplications encompassing one or more exons of PHEX can be analyzed by MLPA. The MLPA reaction was performed according to the standard protocol recommended by the system supplier (Salsa MLPA probemix P223, MRC-Holland, Amsterdam, The Netherlands) (20). The evaluation was carried out with GeneMarker (SoftGenetices, State College, USA).

\section{iPLEX and MALDI-TOF MS}

The mosaic mutation was confirmed by using iPLEX and MALDI-TOF MS (Agena Bioscience, Hamburg, Germany). The iPLEX reaction was performed according to the standard protocol recommended by the system supplier (21). The homogeneous MassEXTEND (hME) and iPLEX process reley on a small volume PCR amplifying the target regions including the SNP position in a multiplex fashion. The basic principle of hME and iPLEX reaction is identical.
Both methods use a third, so-called MassEXTEND primer, which anneals directly adjacent to the SNP position. In an enzymatic primer extension reaction, this primer will be elongated. During that process the allele-specific analytical products are generated. The products differ by mass according to the incorporated bases. Primers were designed: ACGTTGGATGCTGTGAGCACCAATTTGGAC(PHEX-ex21_ PCR1) and ACGTTGGATGTTCTCTTCTAGGTGAGGTGC (PHEX-ex21_PCR2), with the tag-sequences in italics. For the iPLEX-reaction the primer sequences were: ACAGACCAGAAGCTGCC (left) and CCAATTTGGACTTGTTCTC (right). The sample carrier was introduced into the mass spectrometer (MassARRAY Analyzer Compact, Agena Bioscience) and data are fully automatically acquired and analyzed in a real-time setting and revised using Typer software (Agena Bioscience).

\section{Results}

The technical data of the established NGS panel for the molecular confirmation of the diagnosis of XLH are shown in Table 2. Supplementary Sanger sequencing has been established to completely cover sequences with homopolymers in particular. All in all, close to 100\% coverage of all amplicons of the 11 genes in the NGS panel was achieved.

The method proved to be sufficiently robust to process 20 patients in parallel in one reaction approach without loss of quality. 
In DNA samples from 49 patients (patients No. 1-50, except for patient 17) with confirmed XLH, the NGS assay correctly re-identified the PHEX mutations that were already known and classified as pathogenic. Using the NGS method, 15 nonsense, 12 missense, 4 splice, 7 deletions and 4 insertions were found (Supplementary Table 4). For validation, the sequence variants were again confirmed by Sanger sequencing. In one patient, no obvious mutation could be detected by the gene panel. This sample was subsequently also analyzed by MLPA and revealed a duplication of PHEX exon 12, confirming previous results (Supplementary Fig. 1).

DNA samples from three patients that were classified as XLH patients, but in whom no pathogenic mutation in PHEX could be detected beforehand, were also examined with the NGS method (P1-P3; Table $3)$. NGS analysis of the PHEX gene of the first sample strongly indicated a mosaicism at cDNA position 2104 (P1; Table 3). Sixty of 706 reads displayed a $\mathrm{T}$ allele at position c. 2104 while 646 reads displayed the $\mathrm{C}$ allele of the reference sequence (c.2104C>T, C: $91.5 \%$, T: $8.5 \%$ ). This result suggests a mosaic mutation. To confirm the mosaic status in the blood DNA, we verified the NGS analysis employing an assay with iPLEX technology (hME) (Supplementary Fig. 2).

In the last two samples no variation was detected in the PHEX sequence, nor was MLPA suspicious. Therefore, the sequence data of the other genes of the NGS panel were analyzed. The already known nonsense mutation c.31delT (p.Trp11Glyfs*9) in the DMP1 gene was discovered in a homozygous fashion in the first sample (P2; Table 3). In the second sample, two heterozygous missense mutations, c.475C >A (p.Gln159Lys) and c.205A>T (p.Ser69Cys), were discovered in the DMP1 gene. In addition, in this sample three variants were detected in the ENPP1 gene: c.2320C $>\mathrm{T}$ (p.Arg774Cys), and the compound heterozygous mutations c.2662C $>\mathrm{T}$ (p.Arg888Trp) and c. $2663 \mathrm{G}>\mathrm{A}$ (p.Arg888Gln), both affecting codon position 888 (P3; Table 3).

\section{Discussion}

Recently, first international clinical practice recommendations for the diagnosis and management of XLH have been published, recommending that XLH should be diagnosed not only on the basis of clinical signs of rickets and/or osteomalacia in association with renal phosphate wasting, but also on the basis of molecular analysis, confirming the clinical diagnosis on a genetic level (2). Improvements in gene sequencing technologies in combination with rapidly declining costs have led to the development of a large amount of targeted NGS panels. These panels allow investigating multiple known disease-causing genes in one assay. Therefore, in this study, we developed an NGS panel for the diagnosis of XLH and related disorders. We validated the panel for PHEX using 50 DNA-samples with a known PHEX mutation. Since the NGS tool revealed a 100\% agreement in 49 patient samples, the coverage and the sensitivity must be rated very high, proving that we created an easy, fast, and reliable diagnostic tool for the diagnosis of XLH. An exception is large deletions in 46,XX patients or duplications encompassing one or more exons. In these cases, an additional PHEX MLPA analysis must be performed if a mutation cannot be detected with NGS. What sets our XLH gene panel apart from commercial panels is the validation with 50 previously sequenced

Table 3 Variants found in patients 1-3 without proven PHEX-mutation.

\begin{tabular}{|c|c|c|}
\hline Gene & Exon & Variant \\
\hline PHEX & 21 & c. $2104 C=/<T ;$ p. $(\operatorname{Arg} 702 *)$ \\
\hline \multicolumn{3}{|r|}{ 1 } \\
\hline & 2 & c.31delT; p.(Trp11Glyfs*9) \\
\hline & 6 & c.205A>T; p.(Ser69Cys) \\
\hline & 6 & c.475C>A; p.(GIn159Lys) \\
\hline \multicolumn{3}{|r|}{ 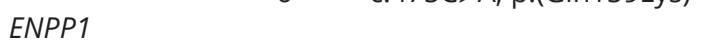 } \\
\hline & 23 & c.2320C>T (p.Arg774Cys) \\
\hline & 25 & c.2662C>T (p.Arg888Trp) \\
\hline & 25 & c.2663G >A (p.Arg888GIn) \\
\hline \multicolumn{3}{|l|}{ FAM20C } \\
\hline & 10 & c.1672C>T (p.Arg558Trp) \\
\hline & 10 & c.1690A>G (p.Asn564Asp) \\
\hline SLC34A3 & 13 & c.1538A>T; p.(Glu513Val) \\
\hline
\end{tabular}

Variant type
Nonsense
Frameshift
Missense
Missense

$\frac{\overline{\text { P1, } \text { ? }}}{\text { Mosaic }}$

P2, ơ

Missense

Missense

Missense

Missense

Missense

Missense

Missense
Mosaic

hom

het

het

het

Compound het Compound het

het

het

het 
DNA samples from clinically confirmed XLH patients. However, the validation could only be carried out for the PHEX gene. There is probably no comparable cohort with already known mutations in the other genes of the panel. Furthermore, due to the high coverage of the genes in the panel we were able to identify a mosaic mutation in the PHEX gene (c.2104C=/<T; p.Arg702*) in one patient sample that had not been detected by Sanger sequencing previously. The reason is most likely that in this case the frequency of the mutated gene copies in DNA from leukocytes was very low (reference base C about 91.5\%, but mutated base $\mathrm{T}$ in only $8.5 \%$ of all reads), but the mosaic mutation has been confirmed by iPLEX and MALDI-TOF MS (Supplementary Fig. 2). Since mosaic mutations are difficult to detect by Sanger sequencing, their description in PHEX is rare in the literature $(22,23,24)$. Therefore, the identification of this mosaic mutation demonstrated the high sensitivity of the developed NGS panel.

However, the advantage of using the panel is not only the molecular diagnosis of XLH, but also of related disorders of renal phosphate wasting in one investigation. For this goal, we included ten other candidate genes in the panel. These genes were decided to be included into our tool, since all these genes are encoding proteins, which are involved in renal phosphate reabsorption and most of them are known to cause a type of renal phosphate wasting disorder in case of a mutation in one of these genes (for details see Table 1 ). A validation of variations in these genes except for PHEX was not possible because of the rarity of these conditions and the unavailability of samples with known mutations.

In one patient, we proved a known homozygous DMP1 mutation, which has been detected before by Sanger sequencing. DMP1 encodes for dentin matrix protein type 1 and is produced by osteoblasts and osteocytes, regulating cell attachment and cell differentiation (25). Homozygous DMP1-mutations are the cause for ARHR type 1 (ARHR1) $(9,10,26)$; a rare autosomal recessive disorder with biochemical and skeletal signs similar to those observed in XLH. Although there are similarities in the pathophysiology between XLH and AHRH1 especially with elevated or inappropriate normal FGF23-levels, the patient can be treated only by the conventional therapy since Burosumab is exclusively licenced for XLH.

In the third sample without proven PHEX mutation, we detected several molecular genetic changes, which could be responsible for the phenotype of the patient. While the DMP1 variants may be common variants, the most probable reason for the phenotype is the compound heterozygous mutations aforementioned in the ENPP1 gene, affecting different positions of the same amino acid codon. Both variants are very rare (allele frequency: $<0.00003$ at gnomAD) (27) and are considered as probably damaging by Polyphen 2 (28) and deleterious by SIFT (sorting intolerant from tolerant) (29). Homozygous or compound heterozygous ENPP1 mutations have been previously described to lead not only to generalized arterial calcification of infancy (GACI1) $(30,31,32)$, but also to autosomal-recessive hypophosphatemic rickets type 2 (ARHR2) in rare cases (8). Although in ARHR2 patients inappropriate high FGF23 levels are seen (8), patients should not be treated with Burosumab, because it is a putative enhancement of the development of vascular calcifications.

Limitations of our approach may be the overall effort that is needed to investigate the genes involved in HR. However, this is reduced if aside from PHEX other genes are included in the NGS analysis. Furthermore, several samples can be studied in parallel, leading to an economic advantage. Moreover, while the overall coverage is quite high, a customized panel can only detect variations in the genes included. Hitherto unknown genes involved in HR will not be investigated. Samples, where no variations are detected with the panel, have to be subjected to other NGS methods with an untargeted approach, namely whole-exome sequencing or even whole-genome sequencing (WGS). These methods have the disadvantage of requiring another, often much more extensive bioinformatics approach for analysis and may be used as a second line. Nevertheless, WGS also offers the possibility to detect structural variations such as inversions (not detectable by MLPA) or deletions by the identification of split-pair reads (33). And lastly, all molecular methods applied will be profitable only in light of very informative patients with respect to clinical and biochemical phenotyping.

In conclusion, the use of NGS technology has major advantages for exact diagnosis of the different forms of HR. In contrast to commercially available NGS panels, the panel was validated with known mutated samples and therefore the application of the panel developed in this study seems to be a sensitive and specific tool which can not only detect mutations in PHEX, but also in other genes associated with HR. This differentiation is favourable for the patients as it readily leads to very specific treatment options.

Supplementary materials

This is linked to the online version of the paper at https://doi.org/10.1530/ EJE-20-0275. 


\section{Declaration of interest}

$\mathrm{O} \mathrm{H}$ and $\mathrm{S} T$ received honoraria from Kyowa Kirin. The other authors have nothing to disclose.

\section{Funding}

This work was in supported by a grant obtained from Kyowa Kirin.

\section{Authors contribution statement}

S Thiele, R Werner, A Stubble, O Hiort and W Hoeppner contributed equally to this research.

\section{References}

1 Beck-Nielsen SS, Brock-Jacobsen B, Gram J, Brixen K \& Jensen TK. Incidence and prevalence of nutritional and hereditary rickets in southern Denmark. European Journal of Endocrinology 2009160 491-497. (https://doi.org/10.1530/EJE-08-0818)

2 Haffner D, Emma F, Eastwood DM, Duplan MB, Bacchetta J, Schnabel D, Wicart P, Bockenhauer D, Santos F, Levtchenko E et al. Clinical practice recommendations for the diagnosis and management of X-linked hypophosphataemia. Nature Reviews: Nephrology 201915 435-455. (https://doi.org/10.1038/s41581-0190152-5)

3 Chesher D, Oddy M, Darbar U, Sayal P, Casey A, Ryan A, Sechi A, Simister C, Waters A, Wedatilake Y et al. Outcome of adult patients with X-linked hypophosphatemia caused by PHEX gene mutations. Journal of Inherited Metabolic Disease 201841 865-876. (https://doi. org/10.1007/s10545-018-0147-6)

4 Saito H, Kusano K, Kinosaki M, Ito H, Hirata M, Segawa H, Miyamoto K \& Fukushima N. Human fibroblast growth factor-23 mutants suppress Na+-dependent phosphate co-transport activity and 1alpha,25-dihydroxyvitamin D3 production. Journal of Biological Chemistry 2003278 2206-2211. (https://doi.org/10.1074/jbc. M207872200)

5 Beck-Nielsen SS, Mughal Z, Haffner D, Nilsson O, Levtchenko E, Ariceta G, de Lucas Collantes C, Schnabel D, Jandhyala R \& Makitie O. FGF23 and its role in X-linked hypophosphatemia-related morbidity. Orphanet Journal of Rare Diseases 201914 58. (https://doi. org/10.1186/s13023-019-1014-8)

6 Imel EA, DiMeglio LA, Hui SL, Carpenter TO \& Econs MJ. Treatment of X-linked hypophosphatemia with calcitriol and phosphate increases circulating fibroblast growth factor 23 concentrations. Journal of Clinical Endocrinology and Metabolism 201095 1846-1850. (https://doi.org/10.1210/jc.2009-1671)

7 Rolvien T, Kornak U, Schinke T, Amling M \& Oheim R. A novel FAM20C mutation causing hypophosphatemic osteomalacia with osteosclerosis (mild Raine syndrome) in an elderly man with spontaneous osteonecrosis of the knee. Osteoporosis International 201930 685-689. (https://doi.org/10.1007/s00198-018-4667-6)

8 Lorenz-Depiereux B, Schnabel D, Tiosano D, Hausler G \& Strom TM. Loss-of-function ENPP1 mutations cause both generalized arterial calcification of infancy and autosomal-recessive hypophosphatemic rickets. American Journal of Human Genetics 201086 267-272. (https://doi.org/10.1016/j.ajhg.2010.01.006)

9 Lorenz-Depiereux B, Bastepe M, Benet-Pages A, Amyere M, Wagenstaller J, Muller-Barth U, Badenhoop K, Kaiser SM, Rittmaster RS, Shlossberg AH et al. DMP1 mutations in autosomal recessive hypophosphatemia implicate a bone matrix protein in the regulation of phosphate homeostasis. Nature Genetics $2006 \mathbf{3 8}$ 1248-1250. (https://doi.org/10.1038/ng1868)
10 Feng JQ, Ward LM, Liu S, Lu Y, Xie Y, Yuan B, Yu X, Rauch F, Davis SI, Zhang $\mathrm{S}$ et al. Loss of DMP1 causes rickets and osteomalacia and identifies a role for osteocytes in mineral metabolism. Nature Genetics 200638 1310-1315. (https://doi.org/10.1038/ng1905)

11 Kruse K, Woelfel D \& Strom TM. Loss of renal phosphate wasting in a child with autosomal dominant hypophosphatemic rickets caused by a FGF23 mutation. Hormone Research 200155 305-308. (https://doi. org/10.1159/000050018)

12 Econs MJ \& McEnery PT. Autosomal dominant hypophosphatemic rickets/osteomalacia: clinical characterization of a novel renal phosphate-wasting disorder. Journal of Clinical Endocrinology and Metabolism 199782 674-681. (https://doi.org/10.1210/ jcem.82.2.3765)

13 Econs MJ, McEnery PT, Lennon F \& Speer MC. Autosomal dominant hypophosphatemic rickets is linked to chromosome 12p13. Journal of Clinical Investigation 1997100 2653-2657. (https://doi.org/10.1172/ JCI119809)

14 Carpenter TO, Whyte MP, Imel EA, Boot AM, Hogler W, Linglart A, Padidela R, Van't Hoff W, Mao M, Chen CY et al. Burosumab therapy in children with X-linked hypophosphatemia. New England Journal of Medicine 2018378 1987-1998. (https://doi.org/10.1056/ NEJMoa1714641)

15 Imel EA, Glorieux FH, Whyte MP, Munns CF, Ward LM, Nilsson O, Simmons JH, Padidela R, Namba N, Cheong HI et al. Burosumab versus conventional therapy in children with $\mathrm{X}$-linked hypophosphataemia: a randomised, active-controlled, open-label, phase 3 trial. Lancet 2019393 2416-2427. (https://doi.org/10.1016/ S0140-6736(19)30654-3)

16 Whyte MP, Carpenter TO, Gottesman GS, Mao M, Skrinar A, San Martin J \& Imel EA. Efficacy and safety of burosumab in children aged 1-4 years with X-linked hypophosphataemia: a multicentre, open-label, phase 2 trial. Lancet: Diabetes and Endocrinology 20197 189-199. (https://doi.org/10.1016/S2213-8587(18)30338-3)

17 Insogna KL, Rauch F, Kamenicky P, Ito N, Kubota T, Nakamura A, Zhang L, Mealiffe M, San Martin J \& Portale AA. Burosumab improved histomorphometric measures of osteomalacia in adults with X-linked hypophosphatemia: a phase 3 , single-arm, International Trial. Journal of Bone and Mineral Research 201934 2183-2191. (https://doi.org/10.1002/jbmr.3843)

18 Kruse K, Hinkel GK \& Griefahn B. Calcium metabolism and growth during early treatment of children with X-linked hypophosphataemic rickets. European Journal of Pediatrics 1998157 894-900. (https://doi.org/10.1007/s004310050962)

19 Rothenbuhler A, Schnabel D, Hogler W \& Linglart A. Diagnosis, treatment-monitoring and follow-up of children and adolescents with X-linked hypophosphatemia (XLH). Metabolism: Clinical and Experimental 2020 103S 153892. (https://doi.org/10.1016/j. metabol.2019.03.009)

20 Schouten JP, McElgunn CJ, Waaijer R, Zwijnenburg D, Diepvens F $\&$ Pals G. Relative quantification of 40 nucleic acid sequences by multiplex ligation-dependent probe amplification. Nucleic Acids Research 200230 e57. (https://doi.org/10.1093/nar/gnf056)

21 Storm N, Darnhofer-Patel B, van den Boom D \& Rodi CP. MALDITOF mass spectrometry-based SNP genotyping. Methods in Molecular Biology 2003212 241-262. (https://doi.org/10.1385/1-59259-327$5: 241)$

22 Goji K, Ozaki K, Sadewa AH, Nishio H \& Matsuo M. Somatic and germline mosaicism for a mutation of the PHEX gene can lead to genetic transmission of X-linked hypophosphatemic rickets that mimics an autosomal dominant trait. Journal of Clinical Endocrinology and Metabolism 200691 365-370. (https://doi.org/10.1210/jc.20051776)

23 Saito T, Nishii Y, Yasuda T, Ito N, Suzuki H, Igarashi T, Fukumoto S \& Fujita T. Familial hypophosphatemic rickets caused by a large deletion in PHEX gene. European Journal of Endocrinology 2009161 647-651. (https://doi.org/10.1530/EJE-09-0261) 
24 Yang M, Kim J, Yang A, Jang J, Jeon TY, Cho SY \& Jin DK. A novel de novo mosaic mutation in PHEX in a Korean patient with hypophosphatemic rickets. Annals of Pediatric Endocrinology and Metabolism 201823 229-234. (https://doi.org/10.6065/ apem.2018.23.4.229)

25 Kalajzic I, Braut A, Guo D, Jiang X, Kronenberg MS, Mina M, Harris MA, Harris SE \& Rowe DW. Dentin matrix protein 1 expression during osteoblastic differentiation, generation of an osteocyte GFP-transgene. Bone 200435 74-82. (https://doi.org/10.1016/j. bone.2004.03.006)

26 Turan S, Aydin C, Bereket A, Akcay T, Guran T, Yaralioglu BA, Bastepe M \& Juppner $\mathrm{H}$. Identification of a novel dentin matrix protein-1 (DMP-1) mutation and dental anomalies in a kindred with autosomal recessive hypophosphatemia. Bone 201046 402-409. (https://doi.org/10.1016/j.bone.2009.09.016)

27 Karczewski KJ, Francioli LC, Tiao G, Cummings BB, Alföldi J, Wang Q, Collins RL, Laricchia KM, Ganna A, Birnbaum DP et al. Variation across 141,456 human exomes and genomes reveals the spectrum of loss-of-function intolerance across human proteincoding genes. bioRxiv 2019. (https://doi.org/10.1101/531210)

28 Sim NL, Kumar P, Hu J, Henikoff S, Schneider G \& Ng PC. SIFT web server: predicting effects of amino acid substitutions on proteins. Nucleic Acids Research 201240 W452-W457. (https://doi. org/10.1093/nar/gks539)

29 Adzhubei IA, Schmidt S, Peshkin L, Ramensky VE, Gerasimova A, Bork P, Kondrashov AS \& Sunyaev SR. A method and server for predicting damaging missense mutations. Nature Methods 20107 248-249. (https://doi.org/10.1038/nmeth0410-248)

30 Cheng KS, Chen MR, Ruf N, Lin SP \& Rutsch F. Generalized arterial calcification of infancy: different clinical courses in two affected siblings. American Journal of Medical Genetics: Part A 2005136 210-213. (https://doi.org/10.1002/ajmg.a.30800)

31 Rutsch F, Boyer P, Nitschke Y, Ruf N, Lorenz-Depierieux B, Wittkampf T, Weissen-Plenz G, Fischer RJ, Mughal Z, Gregory JW et al. Hypophosphatemia, hyperphosphaturia, and bisphosphonate treatment are associated with survival beyond infancy in generalized arterial calcification of infancy. Circulation: Cardiovascular Genetics 20081 133-140. (https://doi.org/10.1161/ CIRCGENETICS.108.797704)

32 Staretz-Chacham O, Shukrun R, Barel O, Pode-Shakked B, Pleniceanu O, Anikster Y, Shalva N, Ferreira CR, Ben-Haim Kadosh A, Richardson J et al. Novel homozygous ENPP1 mutation causes generalized arterial calcifications of infancy, thrombocytopenia, and cardiovascular and central nervous system syndrome. American Journal of Medical Genetics: Part A 2019179 2112-2118. (https://doi. org/10.1002/ajmg.a.61334)

33 dos Santos AP, Meinel J, Piveta CdSC, de Andrade JGR, Fabbri-Scallet H, Gil-da-Silva-Lopes VL, Guerra-Junior G, Kuenstner A, Kaiser FJ, Holterhus P-M et al. Disruption of the topological associated domain at Xp21.2 is related to gonadal dysgenesis: a general mechanism of pathogenesis. medRxiv 2020. (https://doi.org/10.1101/2020.03.25.20041418)

Received 27 March 2020

Revised version received 10 July 2020

Accepted 14 August 2020 